\title{
Incidence of Moderate to Severe Cognitive Dysfunction in Patients Having Carotid Artery Stenting
}

\author{
John G. Gaudet, M.D. ${ }^{1}$, Philip M. Meyers, M.D. ${ }^{2,5}$, James F. McKinsey, M.D. ${ }^{3}$, Sean D. Lavine, \\ M.D. ${ }^{5}$, William Gray, M.D. ${ }^{6}$, Elizabeth Mitchell, B.A. ${ }^{1}$, E. Sander Connolly Jr., M.D. ${ }^{5}$, and Eric \\ J. Heyer, MD, PhD $^{1,4}$ \\ ${ }^{1}$ Department of Anesthesiology, Columbia University, New York, NY \\ ${ }^{2}$ Department of Radiology, Columbia University, New York, NY \\ ${ }^{3}$ Department of Surgery (Division of Vascular Surgery), Columbia University, New York, NY \\ ${ }^{4}$ Department of Neurology, Columbia University, New York, NY \\ ${ }^{5}$ Department of Neurological Surgery, Columbia University, New York, NY \\ ${ }^{6}$ Department of Medicine (Division of Cardiology), Columbia University, New York, NY
}

\begin{abstract}
Introduction-About $25 \%$ of patients with carotid artery stenosis treated with carotid endarterectomy (CEA) develop cognitive dysfunction (CD) 1 day and 1 month after surgery compared to a control group. We hypothesize that patients with carotid artery stenosis treated with carotid artery stenting (CAS) performed under cerebral embolic protection will also develop CD at similar time points when compared to a control group.
\end{abstract}

\begin{abstract}
Methods-Twenty-four patients scheduled for elective CAS were enrolled in a prospective IRB approved study to evaluate cognitive function with a battery of 6 neuropsychometric (NP) tests before, 1 day and 1 month after CAS. Test performance was compared to 23 patients having coronary artery procedures (control group). The mean and standard deviation (SD) of the difference scores in the control group were used to generate z-scores. We used a previously described point system to transform negative z-scores into injury points for each NP test. Global performance is presented as average deficit score (sum of injury points divided by the number of completed tests). All patients underwent these procedures with mild sedation.
\end{abstract}

\section{Results were analyzed in two ways: group-rate analysis and event-rate analysis-}

Outcome was dichotomized by defining moderate to severe cognitive dysfunction as average deficit score at least 1.5 SD worse than the control group. Fisher tests and multivariate logistic regression models were used to analyze group performance.

Results-Control patients tended to be younger and had a lower incidence of stroke or previous transient ischemic attack. One day after surgery, 41\% (10 out of 24) of patients undergoing carotid stenting developed moderate to severe cognitive dysfunction $(\mathrm{p}=0.0422)$. Average deficit score was also significantly higher in the CAS group at 1 day $(\mathrm{p}=0.0265)$. These differences were independent of age and history of stroke/trensiant ischemic attack. Interestingly, we found that the absence of oral statin medication may increase the probability of CD. By 1 month, $9 \%$ (1 out of 11 ) of patient

Corresponding Author and Reprint Requests: Eric J. Heyer, M.D., Ph.D., 630 W 168th St, P\&S Box 46, New York, NY 10032, Phone: 212-305-9072, Fax: 212-305-8287, E-mail: ejh3@columbia.edu.

Conflicts of Interest: None 
undergoing carotid stenting presented with cognitive dysfunction. Other patients were lost to followup.

Conclusion-CAS is associated with a decline in cognitive performance that is at least moderate 1 day after surgery.

\section{Keywords}

Anesthesia; Angioplasty, Transluminal, Percutaneous Coronary; Carotid Arteries; Carotid Stenosis; Cognitive Disorders; Coronary Angiography; Female; Humans; Hydroxymethylglutaryl-CoA Reductase Inhibitors; Male; Monitoring, Intraoperative; Neuropsychological; Tests; Postoperative Complications; Risk Factors; Stents

\section{Introduction}

The prevention of stroke in patients with high-grade carotid artery stenosis is traditionally carried out by carotid endarterectomy (CEA) or more recently carotid artery stenting (CAS). We previously showed (12) that 20 to $30 \%$ of patients undergoing CEA have cognitive dysfunction (CD) between one day and one month postoperatively.

Recent randomized trials indicate that, when compared to CEA, CAS is associated with a higher incidence of stroke or death at 30 days (2). However, neurocognitive evaluations are a more subtle measure of patients' ability to perform complex mental tasks, such as remembering lists of words or copying figures from memory. Using neuropsychometric (NP) tests to measure cognitive performance, changes have been shown to occur after CAS but have not been routinely used to evaluate outcome (reviewed in (1)). In 2000, Crawley et al. published a headto-head comparison of CEA and CAS showing that patients undergoing CAS tended to perform slightly worse on selected cognitive tests (6). Only two prospective studies (23),(15) used control subjects to account for practice effects related to repeated NP testing. Embolic protection devices were not consistently used in these two studies. These authors concluded that cognitive performance either does not change or slightly improves after CAS.

In this prospective observational study, we hypothesized that the incidence of early cognitive dysfunction would be greater in patients undergoing CAS with embolic protection than in a control population undergoing coronary procedures.

\section{Methods}

Population

Twenty-four patients undergoing elective carotid artery stenting (CAS) and 23 control patients undergoing coronary procedures were recruited to participate in this IRB-approved study. We included all patients who were able to complete NP testing in English. Patients were considered for CAS if they met criteria issued by the National Coverage Determination (high risk for CEA presenting with symptomatic carotid artery stenosis $\geq 70 \%$ ), or were at high risk for CEA with either a symptomatic carotid artery stenosis between $50 \%$ and $70 \%$, or an asymptomatic carotid artery stenosis $\geq 80 \%$, in accordance with Category B Investigational Device Exemptions regulations issued by the Food and Drug Administration, patients, could also be considered for CAS. After written, informed consent was obtained, patients were assessed with a battery of NP tests at baseline, one day and one month after surgery. All examinations were performed at least 3 hours after any analgesic or sedative medication had been administered. 


\section{Anesthesia}

Patients were medicated and mildly sedated with midazolam and/or fentanyl during procedures in both groups as needed. However, all patients were able to follow instructions and answer questions during the procedure. For patients undergoing CAS, atropine or robinul were administered to prevent a decline in heart rate during balloon inflation in the carotid artery. Routine hemodynamic and temperature monitoring included arterial blood pressure which was continuously measured through a femoral catheter placed by the surgeon during the procedure. Neurological monitoring was assessed clinically during CAS by having patients answer questions and perform motor tasks.

\section{Procedures}

All coronary procedures were performed by members of the interventional cardiology service. All carotid stenting procedures were performed by members of either the neurovascular service or the vascular service. For CAS procedures, after femoral access was obtained, a diagnostic angiogram was performed in all patients prior to the intervention to confirm the degree of stenosis. Prior to stent placement (ACCULINK ${ }^{*}$ or PRECISE ${ }^{* *}$ ), a protection device (ACCUNET* or ANGIOGUARD ${ }^{* *}$ ) was deployed distal to the stenosis in all cases. Selective carotid angiography was used to guide the procedure. After stent placement and dilation at the lesion site, the local result as well as the distal circulation (to measure changes in flow and screen for possible embolic defects) were assessed. The specific type of stent used for the intervention was left to the discretion of the primary operator or according to protocol. The intervention was considered successful when the residual stenosis was equal or less than $30 \%$. For coronary procedures, femoral access, coronary angiography and/or stenting were performed using standard protocols and materials.

\section{NP evaluation}

Patients were assessed with a battery of NP tests. All examinations were administered by trained research assistants under the supervision of a neuropsychologist. Ten raw scores were generated from a battery of 6 NP tests (Controlled Oral Word Association, Boston Naming, Hopkins Verbal Learning, Rey Complex Figures, Halstead-Reitan Trails A and B, and Grooved Pegboard), which were chosen to represent a range of cognitive domains. For each NP test, the change in performance was calculated by subtracting the baseline score from the postoperative score. The mean change and its standard deviation for patients undergoing coronary stenting (control population) were then used to calculate $z$-scores for each test. A negative value indicates cognitive performance worse than the mean of controls (20). To illustrate cognitive decline, we used a previously described point system to transform negative z-scores into injury points for each NP test (12). As a global measure of cognitive performance, we then calculated an Average Deficit Score (ADS) by dividing the sum of injury points by the total number of completed tests. The ADS maximizes the number of patients included in the analysis by accounting for missing tests; however, it also reduces the effect that single tests have on the overall performance. To compensate for underweighting of specific tests, we considered patients to have moderate to severe cognitive dysfunction if their ADS was greater than the control mean by a minimum of 1.5 standard deviations, and/or z-scores $\leq-1.5$ on two or more tests. We considered patients to have severe cognitive dysfunction if their ADS was greater than the control mean by a minimum of 2 standard deviations, and/or z-scores $\leq-2$ on two or more tests.

\footnotetext{
*Guidant, Abbott vascular, Abbott Park, IL, USA

${ }^{* *}$ Cordis, Johnson\&Johnson, Warren, NJ, USA
} 


\section{Statistics}

Data were stored in a Microsoft Access 2003 database (Microsoft Inc., Redmond, WA) and analyzed with JMP 7.0 (SAS Institute, Cary, NC). All data are presented as mean $\pm 95 \%$ CI or median \pm quartile as appropriate. Normal distribution was tested with the Shapiro-Wilk test.

We used unadjusted nonparametric statistics (Mann-Whitney U test for continuous data, Fisher's exact test for categorical data) to compare outcomes in both groups (CAS and control). The primary outcome was ADS one day and one month after surgery. For all analyses, a twotailed $\mathrm{p}$ value $\leq 0.05$ was considered significant.

Any demographic or surgical data meaningfully correlated with outcome in a univariate analysis (Spearman $\mathrm{p} \leq 0.10$ ) was introduced in a multivariate model for statistical adjustment. Multivariate $\mathrm{p}$ values are presented after verifications of linear assumptions.

\section{Results}

Perioperative variables are summarized in Table 1 . Control patients were, on average, 4 years younger (64.9 vs. 70.3 years, $p=0.0922$ ) and tended to have fewer strokes or transient ischemic attacks (17\% vs. $46 \%, \mathrm{p}=0.0646$ ) (Table 1). Fifty-four percent of the CAS patients were asymptomatic. Amounts of midazolam or fentanyl administered during the procedures were minimal (control vs. carotid: 0.016 vs. $0.012 \mathrm{mg} / \mathrm{kg}$ of midazolam and 0.84 vs. $0.78 \mathrm{mcg} / \mathrm{kg}$ of fentanyl). The median minimal detected stenosis in carotid patients was $90 \%$, ranging from 60 to $100 \%$.

\section{Our results were analyzed in two ways: group-rate analysis and event-rate analysis}

For group-rate analysis, we compared postoperative average deficit scores in both groups. Using nonparametric statistics, median postoperative ADS in the CAS group was significantly higher than in the control group at one day ( 0.6250 in 24 CAS patients, vs. 0.3000 in 23 controls, $\mathrm{p}=0.0265$ ) (Figure 1), suggesting that patients undergoing CAS were relatively more cognitively impaired. Because controls patients were younger and tended to do better neurologically, we introduced these two potential confounders in a multivariate model. The difference remained significant after adjustment for age (multivariate $\mathrm{p}=0.0437$ ) or history of previous stroke or transient ischemic attacks (TIA) (multivariate $\mathrm{p}=0.0453$ ). Therefore, the observed difference in cognitive performance was likely independent of age or neurological history. In CAS patients, neither the type of embolic protection device nor the type of stent was found to influence the outcome.

Results of event-rate analysis are presented in table 3. 10/24 (41\%) patients undergoing CAS and $2 / 23(9 \%)$ control patients presented with $\mathrm{CD}$ that was at least moderate at day 1 postoperatively. This difference was statistically significant and independent of age or history of previous stroke or TIA (Fisher's exact two-tail test, $\mathrm{p}=0.0173$; multivariate $\mathrm{p}=0.0422$ ), suggesting that patients undergoing CAS procedures are exposed to a risk of cognitive dysfunction at day 1 postoperatively that is at least moderate. However, this difference was not statistically significant when considering patients with severe CD only (Fisher's exact twotail test, $\mathrm{p}=0.0971$ ).

Although follow-up cognitive testing at one month was not available in the control group, we assumed that control patients' performance remains stable over the first month. Only $41 \%$ of patients having carotid artery stenting were available at 1 month. Using the same cutoff for definition of $C D$ at one day and one month we identified 1 carotid patient out of $11(9 \%)$ with moderate to severe cognitive dysfunction 1 month postoperatively. Compared to those 11 patients who were successfully evaluated at one month, patients lost to follow-up were sicker ( $71 \%$ versus $36 \%$ of patients ASA status 3 ) but did not perform differently at one day. 
We also looked for risk factors associated with worse cognitive performance in patients undergoing CAS (table 2). Interestingly, all three patients undergoing CAS who were not taking oral statin medications had severe cognitive dysfunction at day 1 postoperatively, whereas only 7 out of $21(33 \%)$ carotid patients taking statins had moderate to severe cognitive dysfunction at day 1 . While all patients taking statins had recovered at one month, 1 out of 3 patients not taking statins remained cognitively impaired.

\section{Discussion}

Using a battery of 6 NP tests to evaluate a range of cognitive domains on a continuous scale (ADS: Average Deficit Score), patients who underwent CAS performed significantly worse than a control population undergoing coronary procedures one day after surgery. This moderate to severe decline in cognitive performance is not due to the effect of anesthesia since patients in both groups were able to follow instructions after receiving minimal sedation.

We recruited patients undergoing coronary procedures as controls in order to account for the effects of drugs administered during conscious sedation, vascular disease, and practice effects associated with repeated NP testing (20). Although globally similar to our study population, control patients were younger and reported a lower incidence of previous stroke or transient ischemic attacks. Age and history of neurological events are two factors known to influence cognitive outcome. Using multivariate regression models to statistically account for the potential influence of each of these two factors, we demonstrate that the observed difference is independent of age or history of neurological events such as stroke or TIA.

We used cognitive change from baseline as a measure of cognitive performance. A control group is needed to determine significant change, i.e. practice effect. However, while 5 investigations conducted from 2000 until 2007 have explored whether carotid artery stenting affects postoperative NP performance (reviewed (1)), only 2 involved a control group (23), (15). A total of 74 carotid patients were enrolled in these two studies. Embolic protection devices were either not used or their use was not reported. They demonstrated that, while verbal memory may transiently improve after carotid stenting, there was no global change at 6 months.

In an attempt to identify patients with cognitive dysfunction we separated patients according to change in cognitive performance. We defined the outcome event of moderate to severe cognitive dysfunction as ADS $\geq 1.5$ standard deviations above the mean of the control group and/or z-scores $\leq-1.5$ on two or more tests. ADS maximizes the number of patients included in the analysis by accounting for missing tests. Although only 47 patients were included in this analysis, we found that 10 carotid patients out of $24(41 \%)$ and 2 control patients out of 23 (9\%) had moderate to severe CD 24 hours after the procedure. Using Fisher's exact two-tail test, this difference proved statistically significant independently of age or history of stroke/ transient ischemic attack.

Our study was insufficiently powered to demonstrate a significant difference in the incidence of severe cognitive dysfunction (ADS $\geq 2 \mathrm{SD}$ above the mean of the control group and/or zscores $\leq-2$ on two or more tests) one day postoperatively. A total of one hundred eighteen patients (59 carotid patients) are needed to show a significant difference in the incidence of severe cognitive dysfunction. We are currently recruiting more patients and hope to verify the hypothesis that the incidence of severe cognitive dysfunction itself is significantly higher following carotid artery stenting procedures.

Control patients were not assessed one month after surgery, but we assumed a stable rate of cognitive dysfunction in this group and used the same cutoff level for definition of $\mathrm{CD}$. While we cannot precisely determine the impact of early dysfunction on late cognitive performance, 
patients appear to improve over time. However, given that more than $50 \%$ of patients were lost to follow-up we cannot make any definitive statement regarding the outcomes at one month.

We believe that cognitive dysfunction at one day expresses true cerebral injury because functional changes, which help compensate for partial cerebral impairment, require more than 24 hours to have any measurable impact. Cognitive performance at one month thus probably reflects how well patients can compensate for subtle cerebral injury. Newman et al., in a longitudinal assessment of neurocognitive function after coronary-artery bypass surgery (18), thus showed that cognitive decline immediately after surgery was significantly associated with both the severity and the incidence of cognitive decline five years later. Most patients in this study did however tend to transiently improve after surgery. At least two mechanisms might underlie the observed improvement in cognitive function between 1 day and 1 month after CAS. First, adjustment of local cerebral perfusion, such as restoration of autoregulation after carotid stenting, is reported to require more than 24 hours to become evident (22). Second, perfusion might also improve with recruitment of new cerebral areas via reversal of "hibernation mechanisms". (10), (21).

The etiology of postoperative cognitive dysfunction in patients undergoing CAS is presumably due to emboli and not hemodynamic effects (9). Multiple groups have documented emboli occurring in CAS by both trans-cranial Doppler (11) and diffusion-weighted MRI (DW-MRI) imaging (13). Carotid protection devices may prevent large emboli from reaching the brain, but micro-emboli cannot effectively be filtered (17). Using DW-MRI after carotid stenting, ElKoussy et al. were able to identify new clinically silent emboli despite the use of a carotid protection device (7).

We have looked for risk factors associated with increased cognitive dysfunction in patients treated with CAS. Despite a small number of patients, we found that the absence of statins significantly increases the probability of CD. At one day, using improvement in blood flow as an outcome, this protective effect is well described in patients undergoing coronary stenting for acute coronary syndrome (3), (16), (14); to our knowledge this would be the first time such an effect is demonstrated in patients undergoing carotid artery stenting (19). Given the limited number of patients included in the analysis, such results should be considered as preliminary. The protective effect of statins might be due to plaque stabilization (5), reduction of systemic inflammation (8), or neuroprotection (4). Other demographic variables frequently correlated with cognitive outcome in patients undergoing carotid endarterectomy (age; diabetes; previous stroke or TIA) were not found to significantly predict the occurrence of cognitive dysfunction in our population.

In conclusion, we demonstrate that CAS procedures are associated with worse cognitive performance one day postoperatively. This decrease in cognitive performance is at least moderate. Despite a significant number of patients lost to follow-up at one month, most CAS patients appear to improve over the first month postoperatively.

\section{Acknowledgments}

Sources of Financial Support:

E.J.H. was supported in part by a grant from the NIA (RO1 AG17604).

J.G.G. was supported in part by a grant from the NIH (T32-GM008464).

Department of Anesthesiology, Columbia University; New York. NY 


\section{Abbreviations}

CEA, Carotid Endarterectomy; CAS, Carotid Artery Stenting; CD, Cognitive Dysfunction; NPM, Neuropsychometric; SD, Standard Deviation.

\section{References}

1. Berman L, Pietrzak RH, Mayes L. Neurocognitive changes after carotid revascularization: a review of the current literature. J Psychosom Res 2007;63:599-612. [PubMed: 18061750]

2. Brahmanandam S, Ding EL, Conte MS, Belkin M, Nguyen LL. Clinical results of carotid artery stenting compared with carotid endarterectomy. J Vasc Surg 2008;47:343-349. [PubMed: 18241758]

3. Celik T, Kursaklioglu H, Iyisoy A, Kose S, Kilic S, Amasyali B, Kardesoglu E, Isik E. The effects of prior use of atorvastatin on coronary blood flow after primary percutaneous coronary intervention in patients presenting with acute myocardial infarction. Coron Artery Dis 2005;16:321-326. [PubMed: 16000891]

4. Cimino M, Balduini W, Carloni S, Gelosa P, Guerrini U, Tremoli E, Sironi L. Neuroprotective effect of simvastatin in stroke: a comparison between adult and neonatal rat models of cerebral ischemia. Neurotoxicology 2005;26:929-933. [PubMed: 15923039]

5. Cimino M, Gelosa P, Gianella A, Nobili E, Tremoli E, Sironi L. Statins: multiple mechanisms of action in the ischemic brain. Neuroscientist 2007;13:208-213. [PubMed: 17519364]

6. Crawley F, Stygall J, Lunn S, Harrison M, Brown MM, Newman S. Comparison of microembolism detected by transcranial Doppler and neuropsychological sequelae of carotid surgery and percutaneous transluminal angioplasty. Stroke 2000;31:1329-1334. [PubMed: 10835452]

7. El-Koussy M, Schroth G, Do DD, Gralla J, Nedeltchev K, von Bredow F, Remonda L, Brekenfeld C. Periprocedural embolic events related to carotid artery stenting detected by diffusion-weighted MRI: comparison between proximal and distal embolus protection devices. J Endovasc Ther 2007;14:293303. [PubMed: 17723007]

8. Gaspardone A, Versaci F. Coronary stenting and inflammation. Am J Cardiol 2005;96:65L-70L.

9. Ghogawala Z, Westerveld M, Amin-Hanjani S. Cognitive outcomes after carotid revascularization: the role of cerebral emboli and hypoperfusion. Neurosurgery 2008;62:385-395. [PubMed: 18382316] discussion 393-385

10. Haubrich C, Kruska W, Diehl RR, Moller-Hartmann W, Klotzsch C. Recovery of the blood pressure - cerebral flow relation after carotid stenting in elderly patients. Acta Neurochir (Wien) 2007;149:131-136. [PubMed: 16964557]discussion 137

11. Hellings WE, Ackerstaff RG, Pasterkamp G, De Vries JP, Moll FL. The carotid atherosclerotic plaque and microembolisation during carotid stenting. J Cardiovasc Surg (Torino) 2006;47:115-126.

12. Heyer EJ, Sharma R, Rampersad A, Winfree CJ, Mack WJ, Solomon RA, Todd GJ, McCormick PC, McMurtry JG, Quest DO, Stern Y, Lazar RM, Connolly ES. A controlled prospective study of neuropsychological dysfunction following carotid endarterectomy. Arch Neurol 2002;59:217-222. [PubMed: 11843692]

13. Kastrup A, Nagele T, Groschel K, Schmidt F, Vogler E, Schulz J, Ernemann U. Incidence of new brain lesions after carotid stenting with and without cerebral protection. Stroke 2006;37:2312-2316. [PubMed: 16888277]

14. Kumar A, Cannon CP. Importance of intensive lipid lowering in acute coronary syndrome and percutaneous coronary intervention. J Interv Cardiol 2007;20:447-457. [PubMed: 18042049]

15. Lehrner J, Willfort A, Mlekusch I, Guttmann G, Minar E, Ahmadi R, Lalouschek W, Deecke L, Lang W. Neuropsychological outcome 6 months after unilateral carotid stenting. J Clin Exp Neuropsychol 2005;27:859-866. [PubMed: 16183618]

16. Longhitano S, Coriat P, Agro F. Postoperative myocardial infarction: pathophysiology, new diagnostic criteria, prevention. Minerva Anestesiol 2006;72:965-983. [PubMed: 17235264]

17. Macdonald S. The evidence for cerebral protection: an analysis and summary of the literature. Eur J Radiol 2006;60:20-25. [PubMed: 16971079] 
18. Newman MF, Kirchner JL, Phillips-Bute B, Gaver V, Grocott H, Jones RH, Mark DB, Reves JG, Blumenthal JA. Longitudinal assessment of neurocognitive function after coronary-artery bypass surgery. N Engl J Med 2001;344:395-402. [PubMed: 11172175]

19. Perler BA. The effect of statin medications on perioperative and long-term outcomes following carotid endarterectomy or stenting. Semin Vasc Surg 2007;20:252-258. [PubMed: 18082842]

20. Rasmussen LS, Siersma VD. Postoperative cognitive dysfunction: true deterioration versus random variation. Acta Anaesthesiol Scand 2004;48:1137-1143. [PubMed: 15352960]

21. Reinhard M, Roth M, Muller T, Guschlbauer B, Timmer J, Czosnyka M, Hetzel A. Effect of carotid endarterectomy or stenting on impairment of dynamic cerebral autoregulation. Stroke 2004;35:13811387. [PubMed: 15087557]

22. van Mook WN, Rennenberg RJ, Schurink GW, van Oostenbrugge RJ, Mess WH, Hofman PA, de Leeuw PW. Cerebral hyperperfusion syndrome. Lancet Neurol 2005;4:877-888. [PubMed: 16297845]

23. Xu G, Liu X, Meyer JS, Yin Q, Zhang R. Cognitive performance after carotid angioplasty and stenting with brain protection devices. Neurol Res 2007;29:251-255. [PubMed: 17178010] 


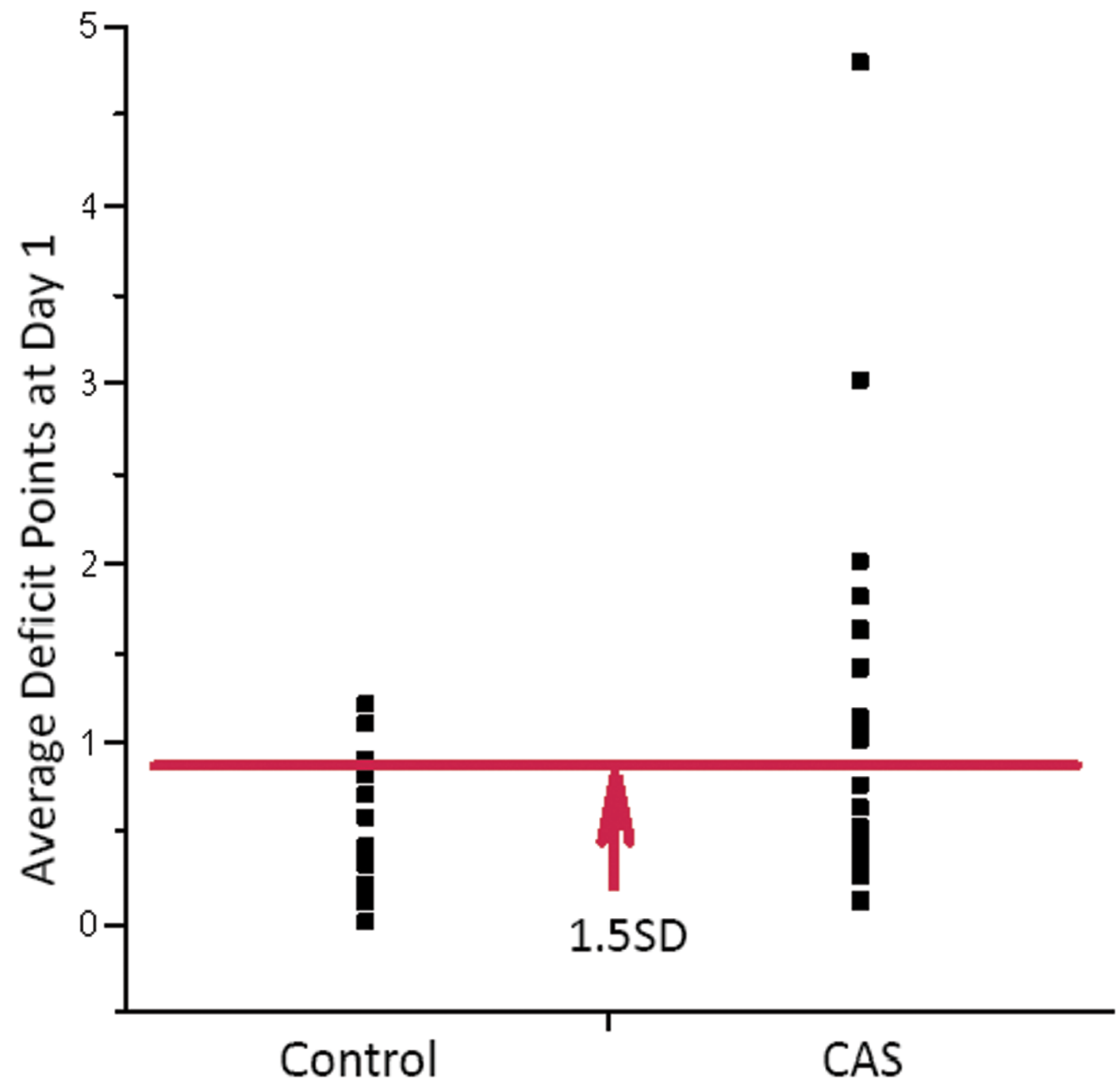

FIGURE 1.

Group-rate analysis at 1 day. Patients undergoing CAS accumulated more deficit points on average at 1 day (non-parametric $\mathrm{p}=0.0265$ ). Points above the red 1.5 SD cutoff line identify patients with moderate to severe cognitive dysfunction. 
TABLE 1

Perioperative characteristics of patients in both groups. Control patients are younger and have a lower incidence of stroke or transient ischemic attack. Analyzes quantifying the relation between group allocation and postoperative cognitive performance are all statistically adjusted for the effect of these two potential confounders

\begin{tabular}{|c|c|c|}
\hline Variable & Controls $(n=23)$ & CAS $(n=24)$ \\
\hline Age [years $\pm 95 \% \mathrm{CI}]$ & $64.9 \pm 4.7$ & $70.3 \pm 4.4$ \\
\hline Gender $[\mathrm{M} / \mathrm{F}]$ & $14 / 9$ & $18 / 7$ \\
\hline Education [years $\pm 95 \% \mathrm{CI}]$ & $15.5 \pm 1.8$ & $14.4 \pm 1.1$ \\
\hline BMI $[\mathrm{kg} / \mathrm{m} 2 \pm 95 \% \mathrm{CI}]$ & $29 \pm 3.1$ & $30.4 \pm 2.9$ \\
\hline Previous stroke or TIA & $4 / 23(17 \%)$ & $11 / 24(46 \%)$ \\
\hline Active smoking & $16 / 23(69 \%)$ & $14 / 24(58 \%)$ \\
\hline Hypertension & $18 / 23(78 \%)$ & $19 / 24(79 \%)$ \\
\hline Diabetes & $7 / 23(30 \%)$ & $10 / 24(42 \%)$ \\
\hline Dyslipidemia & $19 / 23(82 \%)$ & $21 / 24(87 \%)$ \\
\hline Oral statin medication & $20 / 23(87 \%)$ & $22 / 24(92 \%)$ \\
\hline
\end{tabular}


TABLE 2

Perioperative characteristics in carotid patients with and without moderate to severe cognitive dysfunction (CD) at day 1. Older patients not taking statins appeared to be exposed to a higher risk of developing CD at day 1

\begin{tabular}{|c|c|c|}
\hline Variable & CD at day $1(n=10)$ & No CD at day $1(n=14)$ \\
\hline Age & $78 \pm 5$ & $67 \pm 6$ \\
\hline Education & $14 \pm 2$ & $13.5 \pm 3$ \\
\hline BMI & $27.7 \pm 5.1$ & $31.0 \pm 4.1$ \\
\hline Previous stroke or TIA & $5 / 10$ & $6 / 14$ \\
\hline Smoking status & $6 / 10$ & $8 / 14$ \\
\hline Hypertension & $8 / 10$ & $12 / 14$ \\
\hline Diabetes & $3 / 10$ & $6 / 14$ \\
\hline Not taking statins & $3 / 10$ & $0 / 14$ \\
\hline
\end{tabular}




\section{TABLE 3}

Incidence of moderate or severe cognitive dysfunction (CD) at day 1. Moderate CD is defined as ADS greater than the control mean by a minimum of 1.5 standard deviations, and/or z-scores $\leq-1.5$ on two or more tests. Severe CD is defined as ADS greater than the control mean by a minimum of 2 standard deviations, and/or z-scores $\leq-2$ on two or more tests. \# Fisher's exact test

\begin{tabular}{|l|l|l|l|}
\hline Variable & Controls $(\mathbf{n = 2 3})$ & CAS $(\mathbf{n = 2 4})$ & P value \# \\
\hline With moderate CD at day 1 & $2 / 23(9 \%)$ & $10 / 24(41 \%)$ & 0.0173 \\
\hline With severe CD at day 1 & $1 / 23(4 \%)$ & $6 / 24(25 \%)$ & 0.0971 \\
\hline
\end{tabular}

